\title{
CONHECIMENTO DOS RESPONSÁVEIS POR CRIANÇAS DE o A I2 ANOS ATENDIDAS EM UMA UBS DE RECIFE - PE ACERCA DE VIOLÊNCIA INFANTIL
}

\author{
KNOWLEDGE OF RESPONSIBLE FOR CHILDREN FROM o TO I2 YEARS ATTENDED \\ IN A UBS INRECIFE - PE ABOUT CHILD VIOLENCE
}

\author{
Vandreany Cristina da Silva ${ }^{1}$ \\ Nadielle Magdalla Pereira Gomes ${ }^{2}$ \\ Michelle Cardoso Gomes ${ }^{3}$
}

\begin{abstract}
RESUMO: Objetivo: Identificar o nível de conhecimento dos responsáveis de crianças atendidas em uma UBS de Recife-PE sobre violência infantil. Método: Estudo descritivo, transversal, quantitativo. A amostra foi por conveniência e compostapor Ioo responsáveis de crianças atendidas na UBS Professor Romero Marques (Recife - PE). A pesquisa foi aprovada pelo CEP com parecer número 3.306.717. O instrumento utilizado foi um questionário contendo 23 questões, elaborado pelas autoras a partir de referencial teórico acerca do tema. Os resultados foram tabulados e analisados descritivamente com embasamento teórico- científico. Resultados: $60 \%$ da amostra reconhece a violência física como violência infantil, $62 \%$ afirmou não ser necessário bater para educar, $94 \%$ refere que crianças expostas a brigas familiares, tem maior probabilidade de se tornar um adulto agressor; $58 \%$ acredita que a criançaestá mais suscetível a sofrer violência na residência, $45 \%$ considera tapas e beliscões agressões pouco violentas, $85 \%$ entende que estupro é um ato apenas violento, assimcomo murros e chutes (86\%). Conclusão: A violência infantil, apesar de ser um tema bastante explorado, ainda possui muito campo para pesquisa, pois, quando se discuteo entendimento deste conceito com os responsáveis pela criança, ainda se observa muita inconsistência. Os pais, muitas vezes influenciados pela educação que receberam, pelo meio social e pelas influências externas, não veem alguns atos como violência contra a criança. Apesar de leis terem sido introduzidas a fim de melhor esclarecer tais questionamentos, dúvidas e fortalecer o ECA, ainda não há uma estratégia consolidada de educação popular em saúde, ou de políticas que protejam a criança do agressor. Por isto, é necessário que se façam mais estudos neste campoa fim de subsidiar dados para fortalecer e analisar os sistemas de enfrentamento da violência infantil.
\end{abstract}

Descritores: Enfermagem. Saúde da Criança. Saúde da Família. Violência. Infantil. Psicologia Infantil.

ABSTRACT: Objective: To identify the level of knowledge of those responsible for children cared for at a UBS in Recife-PE about child violence. Method: Descriptive, cross-sectional, quantitative

\footnotetext{
${ }^{1}$ Graduada em Enfermagem. Pós- Graduanda em Gestão Hospitalar (UNICAP). Centro Universitário Estácio do Recife.E-mail: Vandreany.cristina@outlook.com.

${ }^{2}$ Bacharel em Enfermagem. Pós-graduanda em Enfermagem do trabalho (FAVENI). Centro Universitário Estácio do Recife.

${ }^{3}$ Enfermeira Especialista em Saúde da Criança UPE. Mestre em Saúde da Criança e do Adolescente UPE. Centro Universitário Estácio do Recife
} 
study. The sample was for convenience and consisted of Ioo guardians of children attended at UBS Professor Romero Marques (Recife - PE). The research was approved by the CEP with opinion number 3,306,717. The instrument used was a questionnaire containing 23 questions, prepared by the authorsfrom a theoretical framework on the subject. The results were tabulated and descriptively analyzed with theoretical-scientific basis. Results: $60 \%$ of the sample recognize physical violence as child violence, $62 \%$ said it is not necessary to hit to educate, $94 \%$ report that children exposed to family quarrels are more likely to becomean aggressor adult; $58 \%$ believe that the child is more likely to suffer violence at home, $45 \%$ consider slapping and pinching as little violent aggression, $85 \%$ understand that rape is just a violent act, as well as punches and kicks (86\%). Conclusion: Child violence, despite being a widely explored topic, still has a lot of research field, because when the understanding of this concept is discussed with those responsible for the child, there is still a lot of inconsistency. Parents, often influenced by the education they received, the social environment and external influences, do not see some acts as violence against the child. Although laws were introduced in order to better clarify such questions, doubts and strengthen the ECA, there is still no consolidated strategy for popular education in health, or policies that protect children from the aggressor. For this reason, it is necessary to carry out more studies in this field in order to subsidize data to strengthen and analyze the systems for fighting violence against children.

Keywords: Nursing; Child Health. Family Health. Child Violence. Child psychology.

\section{INTRODUÇÃO}

A violência infantil ainda é uma realidade observada em diferentes contextos, trazendo impacto negativo para o crescimento e desenvolvimento da criança e pode estar relacionado às diversas formas de negligência, abuso e maus-tratos. A caracterização da violência contra a criança é compreendida além da agressão física e psicológica, mas também por atos de omissão e negação dos diretos da criança, conforme preconiza o Estatuto da Criança e do Adolescente (ECA) (EGRY; APOSTOLICO; MORAIS, 2017).

Segundo o Ministério da Saúde (MS), entre 20II a 2017, o Sistema de Informação de Agravos de Notificação (SINAN), registrou 1.460 .326 casos de violência no Brasil, sendo 219.717 somente contra criança e 372.014 contra adolescentes, somando um total de $40,5 \%$ dos casos notificados nesses dois períodos da vida. Entre os anos de 2015 e 2016 o Disque roo recebeu $37 \mathrm{mil}$ denúncias associadas a atos de violência contra pessoas menores de 18 anos, sendo a maioria do sexo feminino, contabilizando um número alarmante de denúncias referentes àviolência sexual (DESLANDES; MENDES; LUZ, 2014).

De acordo com o Estatuto da Criança e do Adolescente (ECA) considera-se criança toda pessoa que possua até doze anos incompletos de idade, classificando esse período como infância. A Organização Pan-Americana de Saúde (OPAS) enfatiza o compromisso mundial de investir no desenvolvimento infantil logo na primeira infância. Os cuidados nesses primeiros anos de vida são decisivos no desenvolvimento cognitivo, emocional e social, garantindo resultados positivos ou negativos ao longo da vida (YAKUWA; NEILL; MELLO, 2018).

A violência pode ser identificada em dois aspectos, a violência física e/ou do poder, podendo ser utilizada para si própria ou para o outro. A Organização Mundial de Saúde (OMS) classifica violência em quatro tipos sendo elas: física, psicológica, sexual e a negligência que, apesar de sua difícil definição, acarreta em danos físicos e psicológicos 
irreversíveis na vida da criança, sendo ainda a forma mais frequente no mundo (MAGALHÃES et al., 2017).

As consequências da violência irão repercutir tanto na vida adulta quanto na criança durante seu processo de desenvolvimento, resultando em desvios de conduta, transgressão a regras sociais, entre outros distúrbios psicossociais que resultarão em condição de risco para o indivíduo e para sociedade (PASIAN et al., 2014). Observam-se também as consequências de violência por negligência evidenciada pela ausência de cuidados de higiene, moradia, alimentação e saúde, resultando em baixo peso e imunidade sensibilizada, por exemplo, favorecendo o aparecimento de doenças oportunista (EGRY; 2015).

No ECA pode-se encontrar diversas diretrizes criadas para melhor atender essa população, garantindo a promoção da saúde e proteção física e social. A partir dele, o Brasil avançou no que diz respeito às investigações e maior número de denúncias de maus-tratos. O aspecto legal preconizado sobre violência infantil torna obrigatória a identificação e a denúncia de violência contra a criança, mesmo em casos de suspeita (MOREIRA et al., 2014).

Mesmo assim, muitos responsáveis por crianças não reconhecem alguns comportamentos como violência. A falta de estrutura familiar somada a outros fatores externos aparece como principais influenciadores para o agravo da situação pode-sedestacar entre tantos fatores, a omissão da instância pública, baixo grau de escolaridade, situação socioeconômica, abandono, desemprego e a pouca idade dos responsáveis legais (NUNES et al., 2013).

$\mathrm{O}$ acolhimento das vítimas deve ser um processo intersetorial e não deve ficar a

cargo apenas dos profissionais de saúde. O trabalho em conjunto dos profissionais envolvidos deve ter critérios para uma melhor qualidade da assistência, assistindo integralmente a demanda existente, agindo em sua totalidade diretamente com o Sistema Único de Saúde (SUS) que oferece o acolhimento em todas as esferas necessárias (TEIXEIRA; COUTO; DELGADO, 2017).

A Enfermagem é fundamental no processo de acolhimento a violência infantil, tendo autonomia para intervir e fornecer assistência legal necessária a essa vítima. $O$ primeiro atendimento às vítimas é de suma importância na tomada das próximas decisões, por isso, é necessário preparação e conhecimento no que diz respeito ao assunto. $\mathrm{O}$ enfermeiro precisa estar atento às necessidades da vítima e preparado para o acolhimento integral, disponível para ouvir e apoiar a vítima, de maneira que não julgue, mas busque compreender as dificuldades enfrentadas e as razões pelas quais foi submetida a esse processo (EGRY; 2018).

Objetivou-se identificar o nível de conhecimento dos responsáveis de crianças atendidas em uma UBS de Recife - PE sobre violência infantil.

\section{MATERIAL E MÉTODO}

Trata- se de um estudo do tipo descritivo, de corte transversal, com abordagem quantitativa. A coleta de dados foi realizada no período de abril e maio de 2019 na UBS Professor Romero Marques, após aprovação do estudo pelo CEP do Centro Universitário 
Estácio do Recife com parecer: 3.306.717 e CAAE: 10637619.8.00oo.5640. O instrumento utilizado para coleta de dados da pesquisa foi um questionário contendo 23 questões, elaborado pelas autoras a partir de referencialteórico acerca do tema. Os resultados foram tabulados e apresentados através de dados estatísticos simples de frequência absoluta e relativa em forma de tabelas e/ou gráficos e foram analisados descritivamente com embasamento teórico- científico.

\section{RESULTADOS E DISCUSSÃO}

A tabela I apresenta a relação dos dados sociodemográficos da amostra do estudo, sendo, $76 \%$ foi do sexo feminino, $27 \%$ tinham idade variando entre 35 a 45 anos, $60 \%$ eram solteiros e $38 \%$ eram praticantes do catolicismo; $68 \%$ possuía rendafamiliar de um salário mínimo, 67\% tinha ensino fundamental incompleto e $50 \%$ da amostra trabalhava informalmente.

Para a OMS (2017), a violência infantil está diretamente ligada aos fatores socioeconômicos, tais como renda familiar, grau de escolaridade e classe social. A amostra do presente estudo é caracterizada, de forma geral, por indivíduos de baixo nível socioeconômico, e, consequentemente, de menor entendimento do conceito de violência contra a criança. A baixa escolaridade, associada à baixa renda, favorece atos violentos no meio familiar, não somente contra a criança.

Tabela r. Perfil sociodemográfico da amostra, Recife-PE, 2019.

\begin{tabular}{|c|c|c|}
\hline VARIÁVEL & $\mathbf{N}$ & $\%$ \\
\hline \multicolumn{3}{|l|}{ SEXO } \\
\hline Masculino & 24 & 24 \\
\hline Feminino & 76 & 76 \\
\hline \multicolumn{3}{|l|}{ IDADE } \\
\hline$<\mathrm{I} 8$ anos & 25 & 25 \\
\hline 18 a 35 anos & 32 & 32 \\
\hline$>35$ anos & 43 & 43 \\
\hline \multicolumn{3}{|l|}{ ESTADO CIVIL } \\
\hline Solteiro & 70 & 70 \\
\hline Casado & 19 & 19 \\
\hline Viúvo & 2 & 2 \\
\hline União Estável & 9 & 9 \\
\hline \multicolumn{3}{|l|}{ RELIGIÃO } \\
\hline Católico & $4 \mathrm{I}$ & $4 \mathrm{I}$ \\
\hline Protestante & 38 & 38 \\
\hline Espírita & 8 & 8 \\
\hline Outros (Ateu, Religiões de Matriz Africana) & 13 & 13 \\
\hline RENDA FAMILIAR & & \\
\hline
\end{tabular}




\begin{tabular}{|c|c|c|}
\hline Até I Salário & 68 & 68 \\
\hline De I a 2 Salários & 27 & 27 \\
\hline Sem Renda & 5 & 5 \\
\hline \multicolumn{3}{|l|}{ Grau de escolaridade } \\
\hline Ensino Fundamental Completo & Iо & Iо \\
\hline Ensino Fundamental Incompleto & 67 & 67 \\
\hline Ensino Médio Completo & $2 \mathrm{I}$ & $2 \mathrm{I}$ \\
\hline Ensino Superior & 2 & 2 \\
\hline \multicolumn{3}{|l|}{ Situação Atual de Trabalho } \\
\hline Trabalha de Carteira Assinada & 18 & 18 \\
\hline Trabalhos Informais & 50 & 50 \\
\hline Não Trabalha & 32 & 32 \\
\hline \multicolumn{3}{|c|}{ GRAU DE PARENTESCO DO RESPONSÁVEL } \\
\hline Mãe & 68 & 68 \\
\hline Pai & 9 & 9 \\
\hline Avós & 14 & 14 \\
\hline Cuidadores & 9 & 9 \\
\hline Total & 100 & 100 \\
\hline
\end{tabular}

Fonte: dados da pesquisa

Destaca-se aqui o papel da mãe como principal cuidadora da criança (68\%). Papel este que, historicamente, ainda é incumbido à mulher. Isso se deve ao determinismo social imposto em que a mulher ainda é inferiorizada para a realização de determinados processos, assumindo assim, o papel de cuidadora do lar, apropriando-se de atividades domésticas como lavar, cozinhar e limpar, além do cuidado com os filhos e marido preocupando-se com educação, higiene, alimentação e saúde. A autoria dos "abusos", se dá em sua maioria, aos responsáveis pela criança, pois, dedica maior parte do tempo à mesma. Os problemas conjugais, financeiros e o desemprego são as principais causas de estresse dentro das casas, o cuidador acaba descontando suas frustações e impaciência na criança, seja em forma de desprezo ou agressão (LOPES; ZANON, 2014).

A literatura relata que a maioria dos agressores, vive ou viveu algum tipo de agressão ainda na infância, seja ela psicológica, física ou sexual. Esse fator é de extrema importância, pois é comprovado que alguém que sofreu violência infantil, na vida adulta terá maiores chances de continuar a propagar a violência no meio intrafamiliar. Isto explica-se pela cultura do meio social no qual está inserido (PATIAS; BOSSI, 2014).

A tabela 2 apresenta os resultados em relação ao sexo e idade da criança e se a mesma frequenta a escola. Observa-se que $57,3 \%$ são meninos, na faixa etária entre 2 e 5 anos (32\%). A literatura aponta que, embora a violência infantil ainda não tenha definições concretas, por parte dos agressores, as crianças menores de cinco anos são as mais acometidas. Isto é explicado devido à maior vulnerabilidade nesse período da infância. Por isto, torna-se 
importante esclarecer para os responsáveis da amostra o conceito e os tipos de violência que podem ocorrer, mesmo que os mesmosacreditem que algumas ações não sejam atos violentos (BESERRA et al., 2013).

Verifica-se ainda na tabela 2 que $69 \%$ frequenta a escola. É de granderelevância a participação da criança na escola, pois as crianças com idade entre 2 e 5 anos estão em um período de descobertas e amplo desenvolvimento social. A escola possui um ambiente propício para o estímulo de novas descobertas, criatividade individual e percepção de fatos. Além disso, tem um efeito positivo na proteção e conscientização das crianças e das famílias sobre a violência infantil, uma vez que, em algumas situações, é na escola que se percebe que a criança sofre violência (SANTOS et al., 2018).

Tabela 2. Perfil sociodemográfico das crianças, Recife-PE, 2019.

\begin{tabular}{lccc}
\hline & VARIÁVEL & $\mathrm{N}$ & \\
\hline SEXO DA CRIANÇA & & & \\
Feminino & 29 & 42,64 \\
Masculino & 39 & 57,36
\end{tabular}

\section{IDADE DA CRIANÇA}

\begin{tabular}{lcc} 
Entre o e 2 anos & 27 & 27 \\
Entre 2 e 5 anos & 32 & 32 \\
Entre 5 e Io anos & 22 & 22 \\
Entre Io e I2 anos & 19 & 19 \\
A CRIANÇA FREQUENTA & 69 & 69 \\
Escola & 19 & 19 \\
Creche & 12 & 12 \\
Não Frequenta & 100 & 100 \\
\hline TOTAL & 12 &
\end{tabular}

Fonte: dados da pesquisa

A tabela 3 apresenta a opinião dos responsáveis sobre questões relacionadas a violência contra acriança, onde apenas $34,30 \%$ reconhece a violência física como violência infantil. De acordo com a OMS define-se violência como o uso da força físicaou poder, para ameaçar outra pessoa ou a si próprio. No entanto, apenas uma parte da amostra teve este entendimento. (GOVINDAMA, 2015). 
Tabela 3. Distribuição da a amostra do estudo quanto ao conhecimento dos responsáveis acerca daviolência contra a criança, Recife-PE, 2019.

\begin{tabular}{lcc}
\hline \multicolumn{1}{c}{ VARIÁVEL } & $\mathrm{N}$ & $\%$ \\
\hline QUAIS TIPOS DE VIOLÊNCIA CONHECE & & \\
Violência Sexual & 51 & 29,14 \\
Violência Física & 60 & 34,30 \\
Negligência & 12 & 6,85 \\
Violência Psicológica & 52 & 29,71 \\
É NECESSÁRIO BATER PARA EDUCAR & & \\
Sim & 38 & 38 \\
Não & 62 & 62
\end{tabular}

CRIANÇAS EXPOSTAS A BRIGAS SOFREM INTERFERÊNCIA NODESENVOLVIMENTO

$\operatorname{Sim}$

Não

LOCAL ONDE A CRIANÇA É MAIS EXPOSTA A VIOLÊNCIA

Casa

Escola

Rua

JÁ OUVIU FALAR NA LEI DA PALMADA

Sim

Não

ENTENDE O SIGNIFICADO DE VIOLÊNCIA INFANTIL

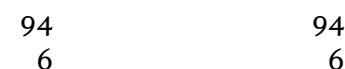

$58-58$

$16-16$

$26 \quad 26$

$44 \quad 44$

\begin{tabular}{lll} 
Sim & 100 & 100 \\
\hline Total & 100 & 100
\end{tabular}

Fonte: dados da pesquisa

No entanto, verifica-se que $62 \%$ diz que não é necessário bater para educar, e, $38 \%$ da amostra diz que é de extrema necessidade uma "palmada de vez e quando" considerando que essa palmada o tornará um indivíduo melhor no futuro. A palmada,por ser a agressão mais popular dentre as outras, não é considerada violência pelos responsáveis, essa prática vem sendo executada desde a descoberta do Brasil, os jesuítas incluíram a palmada no processo educacional de crianças que perdurou por mais de 200 anos, até serem expulsos pelo Marquês de Pombal em 176o, porém os castigos físicos ás crianças já estavam introduzidos no meio intrafamiliar e mantem- se até hoje passando de pai para filho (PATIAS; 2012).

Apesar de 56\% diz saber o que significa a Lei da Palmada, não se sentiram à vontade para descrever tal conhecimento, e, todos afirmam saber o significado de violência infantil. A Lei 13.010 , de 26 de junho de 2014, também chamada de "Lei daPalmada", foi criada com a finalidade de proteger crianças e adolescentes, a fim de garantir seus direitos e diminuir agressões físicas e, posteriormente, psicológicas. Causou muita polêmica entre os pais e responsáveis desse público alvo, pois a palmada tornou-se um ato banalizado e rotineiro, sendo conhecido pelos responsáveisum método pedagógico (SAYÃO; 2014). 
Quanto aos sinais e consequências da violência infantil, verificou-se que 30,9\% da amostra afirma que o principal indicador apresentado pela criança é a agressividade, $90 \%$ diz que a criança vítima de violência terá mais probabilidade de se tornar um adulto agressor e $69 \%$ acredita que o sexo praticado com crianças maiores de 12 anos é violência sexual, mesmo com o consentimento da mesma (dados não demonstrados)

Os traumas vivenciados pelas crianças acarretam em diversos transtornos sociais, psicológicos e físicos. Quadros de ansiedade, demonstração de insatisfação com a vida, sobrepeso, ideação suicida, enxaquecas, automutilação, agressividade e autoritarismo são algumas das características observadas em crianças que são ou foram vítimas de atos de violência. As experiências negativas ao decorrer do seu crescimento e desenvolvimento originam resultados nocivos dentro do que se conceitua moral e ético na sociedade (DESLANDES; MENDES; PINTO, 2015).

A maioria da amostra $(88,0 \%)$ referiu conhecer o ECA, mas não sabe referir sua importância e finalidade (dados não demonstrados). Mesmo após a criação do (ECA) em 1990, que abrange o nível de proteção contra crianças de o a il anos incompletas e garante sua defesa, nota-se que o índice de violência continua latente de forma oculta e omissa, principalmente no ambiente familiar. Os pais/responsáveis não entendem e/ou não têm o concreto conhecimento que estão sendo destruidores de valores e, agem assim naturalmente, a fim de obter obediência dos filhos (PASIAN et al., 2015).

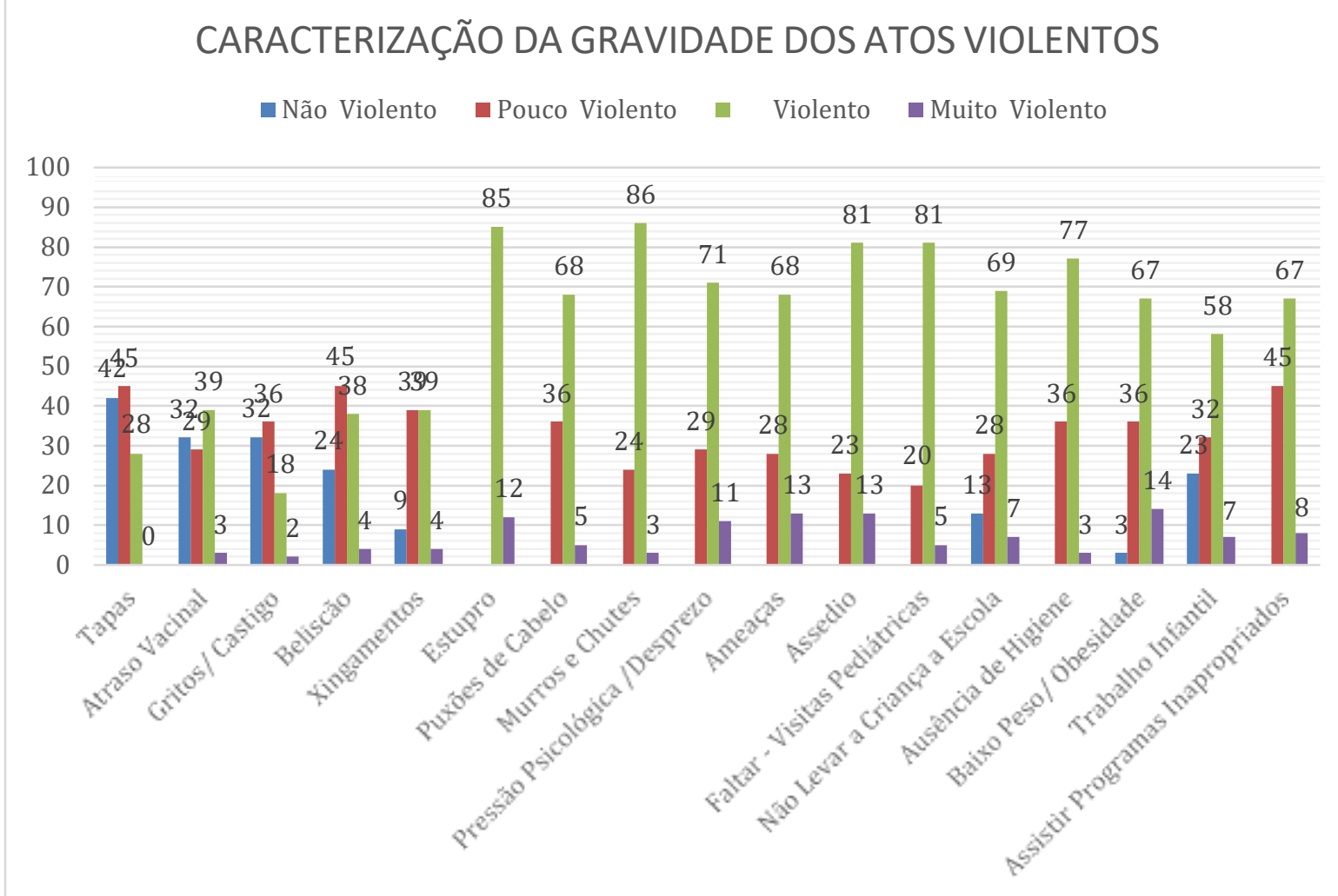

Gráfico I Distribuição da a amostra do estudo quanto à caracterização da violência contra a criança, Recife- 
PE, 2019.

De acordo com resultados apresentados no gráfico I, 45 indivíduos da amostra considera tapas e beliscões agressões pouco violentas; 85 entende que estupro é um ato apenas violento; 86 afirmaram que murros e chutes são atitudes violentas; e, ainda 8I consideraram o assédio um ato violento e esse mesmo quantitativo concorda que faltar visitas pediátricas também é um ato violento.

A violência certamente sempre fez parte do cotidiano dos seres humanos. O problema é que essa banalização afeta de modo importante a vida das crianças, por serem mais frágeis e propensos a sofrerem violência, seja por estranhos, seja por familiares. A banalização cotidiana dos tapas e palmadas ainda está intrínseca na estrutura familiar da maioria da população, prática esta que precisa ser revista e amplamente combatida. Infelizmente, somente os atos mais agressivos são considerados violentos pelos responsáveis, talvez fruto da construção histórica da sociedade, principalmente entre os menos favorecidos (MOURA; OLIVEIRA; VASCONCELOS, 2015).

\section{CONCLUSÕES}

A violência infantil, apesar de ser um tema bastante explorado, ainda possui muito campo para pesquisa, pois, quando se discute o entendimento deste conceito com os responsáveis pela criança, ainda se observa muita inconsistência. Os pais, muitas vezes influenciados pela educação que receberam, pelo meio social e pelas influências externas, não veem alguns atos como violência contra a criança.

A violência física contra a criança foi motivo de grande discussão e não concordância durante a coleta de dados, pois muitas atitudes tomadas eram consideradas formas de disciplinar, já para outros eram tidas como um tipo de violência grave.

Apesar de leis terem sido introduzidas a fim de melhor esclarecer tais questionamentos, dúvidas e fortalecer o ECA, ainda não há uma estratégia consolidada de educação popular em saúde, ou de políticas que protejam a criança do agressor. Por isto, é necessário que se façam mais estudos neste campo a fim desubsidiar dados para fortalecer e analisar os sistemas de enfrentamento da violênciainfantil.

\section{REFERÊNCIAS}

BESERRA, Maria Aparecida et al. Prevalência de violência na escola e uso de álcool e outras drogas entre adolescentes. Rev. Latino-Am. Enfermagem, Ribeirão Preto, v.27, e3Iro, 2013.

DESLANDES, S.F; MENDES, C.H.F; LUZ, E.S. Análise de desempenho de sistema de indicadores para o enfrentamento da violência intrafamiliar e exploração sexual decrianças e adolescentes. Ciências saúde coletiva, Rio de Janeiro, v. I9, n. 3, p. 865- 874, 2014. 
DESLANDES, Suely; MENDES, Corina Helena Figueira; PINTO, Liana Wernersbach. Proposição de um índice do enfrentamento governamental à violência intrafamiliar contra crianças e adolescentes. Caderno Saúde Pública, Rio de Janeiro, v. 31, n. 8, p. 1709-1720, ago. 2015 .

EGRY, E.Y et al. Enfrentar a violência infantil na Atenção Básica: como os profissionais percebem? Revista Brasileira Enfermagem, Brasília, v. 70, n. I, p. I19- I25, 2017.

EGRY, E.Y et al. Entendendo a negligência infantil em um contexto de gênero: um estudo realizado em uma cidade brasileira. Revista Escola Enfermagem. USP, São Paulo, v. 49, n. 4, p. 0556-0563, 2015.

EGRY, E.Y; APOSTOLICO, M.R; MORAIS, T.C.P. Notificação da violência infantil, fluxos de atenção e processo de trabalho dos profissionais da Atenção Primária em Saúde. Ciênc. saúde coletiva, Rio de Janeiro, v. 23, n. I, p. 83-92, 2018

GOVINDAMA, Y. O sofrimento da criança pequena maltratada e seus pais. Estilos clínicos, São Paulo, v. 20, n. I, p. 43-58, 2015.

LOPES, Manuela Nunes; DELLAZZANA-ZANON, Letícia Lovato; BOECKEL, Mariana Gonçalves. A multiplicidade de papéis da mulher contemporânea e a maternidade tardia. Temas psicológicos, Ribeirão Preto, v. 22, n. 4, p. 917-928, dez. 2014.

MAGALHAES, J.R. F et al. Expressão da violência intrafamiliar: História oral de adolescentes. Texto contexto - enfermagem., Florianópolis, v. 26, n. 4, er730or6, 2017.

MOREIRA, G.A. R et al. Fatores associados à notificação de maus-tratos em crianças e adolescentes na atenção básica. Ciências saúde coletiva, Rio de Janeiro, v. 19, n. Io, p. 42674276, 2014 .

MOURA, L.B; OLIVEIRA, C; VASCONCELOS, A.M.N. Violências e juventude em um território da Área Metropolitana de Brasília, Brasil: uma abordagem sócio espacial. Ciênc. saúde coletiva, Rio de Janeiro, v. 20, n. II, p. 3395-3405, 2015.

NUNES, A.J; SALES, M.C.V. Violência contra crianças no cenário brasileiro. Ciências saúde coletiva, Rio de Janeiro, v. 2I, n. 3, p. 871-88o, 2016.PASIAN, M.S. et al. Negligência infantil: a modalidade mais recorrente de maus-tratos. Pensando fam., Porto Alegre, v. I7, n. 2, p. 6i-7o, 2016.

PASIAN, M.S. et al. Negligência infantil a partir do Child Neglect Index aplicado no Brasil. Psicologia Reflexiva Critica, Porto Alegre, v. 28, n. I, p. I06-II5, 2015.

PATIAS, Naiana Dapieve; BOSSI, Tatiele Jacques; DELL'AGLIO, Débora Dalbosco. Repercussões da exposição à violência conjugal nas características emocionais dos filhos: revisão sistemática da literatura. Temas psicológicos, Ribeirão Preto, v. 22, n. 4, p. 901-915, 
dez. 2014.

PATIAS, Naiana Dapieve; SIQUEIRA, Aline Cardoso; DIAS, Ana Cristina Garcia. Bater não educa ninguém! práticas educativas parentais coercitivas e suas repercussões nocontexto escolar. Educação Pesquisa, São Paulo, v. 38, n. 4, p. 981-996, Dec. 2012.

SANTOS, Marconi de Jesus et al. Caracterização da violência sexual contra crianças e adolescentes na escola - Brasil, 20ı-2014. Epidemiologia Serviço Saúde, Brasília,v. 27, n. 2, e2017059, 2018.

SAỸ̃O, R. Palmada Educa ou Deseduca. Folha de S. Paulo. o7 de abril de 2005. Disponível em: Acesso em: I8 nov. 2014.

TEIXEIRA, Melissa Ribeiro; COUTO, Maria Cristina Ventura; DELGADO, Pedro Gabriel Godinho. Atenção básica e cuidada colaborativo na atenção psicossocial de crianças e adolescentes: facilitadores e barreiras. Ciências saúde coletiva, Rio de Janeiro, v. 22, n. 6, p. 1933-1942, jun. 2017. 